\title{
Performance Benefits of Spatially Distributed Versus Stacked Information on Integration Tasks
}

\author{
JOOYOUNG JANG* and CHRISTIAN D. SCHUNN
}

Learning Research and Development Center, University of Pittsburgh, Pittsburgh, USA

\begin{abstract}
Summary: Understanding the best way to present information has been an issue of interest to many researchers. Regardless of the content of the information, purely physical elements such as spatial organization may also influence performance. Across two studies with 111 undergraduates (78 in Study 1 and 33 in Study 2), we compared spatially distributed (i.e. when information sources are presented side-by-side) against stacked displays (i.e. when information sources are sitting on top of one another with only the top source fully visible). A distributed display time advantage was consistently observed. As potential explanations, a memory strategy selection hypothesis was examined along with other procedural alternative explanations. Copyright $\odot 2011$ John Wiley \& Sons, Ltd.
\end{abstract}

Some tasks require processing single information sources in isolation (e.g. simple games, texting), but many tasks involve integrating multiple sources of information. Saliently then for the design of displays is the question of how to spatially organize multiple sources of information in such integrative tasks to support information integration during problem solving. Prior aviation and education research lines have examined approaches to display information integration and their effects on learning and task performance. These researchers argued that displays should spatially integrate information that needs to be mentally integrated. We build on this work to examine spatial distribution.

Cognitive load theory (Sweller \& Chandler, 1994; Sweller, van Merriënboer, \& Paas, 1998; van Merriënboer \& Sweller, 2005) suggests that instructional materials should reduce extraneous cognitive load. One concrete strategy involves 'replacing multiple sources of information (frequently pictures and accompanying text) with a single, integrated source of information' (van Merriënboer \& Sweller, 2005, p. 151). Information integration tasks involve high cognitive load from the core task, and therefore, they especially benefit from low cognitive load imposed by the interface. Integrated display removes the cognitive load imposed by the interface to find and mentally integrate multiple information sources.

Within human factors research on interactions between task demands and information display layout (Kroft \& Wickens, 2002; Wickens \& Carswell, 1995; Wickens \& McCarley, 2008), the proximity compatibility principle has been proposed. This design principle recommends high perceptual proximity when processing proximity is high (i.e. when information sources need to be integrated). Perceptual proximity can be achieved by placing sources at a close distance or by grouping sources using common visual features such as color or orientation.

Kroft and Wickens (2002) tested various forms of integrative displays. In particular, they had pilots use six different display versions: four integrated displays differentiated by the manner of integration (e.g. a large integrated display and a small integrated display) and two

\footnotetext{
*Correspondence to: Jooyoung Jang, Department of Psychology, University of Pittsburgh, 823 LRDC (Learning Research and Development Center), 3939 O'Hara Street, Pittsburgh, PA 15260 USA.

E-mail: joj15@pitt.edu
}

separated displays (i.e. a small separated side-by-side display and a large separated display, only one source visible). Overall, pilots answered questions faster and more accurately when the sources were integrated but only for questions that demanded integration across the information sources.

A different but closely related aspect of display organization that can support integrative tasks involves spatially distributed versus stacked displays. ${ }^{1}$ For example, multiple graphs can be displayed side-by-side or they can sit on top of another. As another example, large graphs can be printed out and spread out on a table or they can be examined one at a time on a monitor. For many complex tasks (e.g. weather forecasting, aviation, geoscience), it is not possible to integrate all relevant task information into one readable display, and thus, the distributed versus stacked information display issue is a very broadly applicable factor. This contrast of spatial organization is different from the integrated versus separated contrast (see Table 1). ${ }^{2}$

Do the same theories/principles apply to the case of distributed versus stacked displays? Initially, we examine empirical evidence for the effect of spatially distributed versus stacked displays. Then, we will return to theories that might explain the effect.

\section{The effect of distributed versus stacked displays}

Trafton, Trickett, Schunn, and Kirschenbaum (2007) investigated complex visualization usage by intermediate meteorological forecasters. They observed a surprising and large time difference to complete the task with a computer

\footnotetext{
${ }^{1}$ The two displays are labeled to emphasize their difference in spatial organization. But one could conceive of spatially distributed displays as also integrated in time (i.e. all sources presented simultaneously), whereas stacked displays are distributed in time.

${ }^{2}$ Although Kroft and Wickens (2002) examined two separated formats that were compatible to spatially distributed (small separated, in authors' term) and stacked displays (interactive decluttering, in authors' term), the conditions were not directly compared as it was not the focus of their study. Their data suggest mixed effects between the two conditions (with no information presented regarding the statistical significance of these comparisons); about 1 second faster reaction time observed in stacked display for questions requiring focused attention, similar reaction time across both displays for questions requiring divided attention, and a null effect for overall accuracy $(\sim 85 \%)$.
} 
Table 1. A schematic comparison for display formats

Sntegrative display
Separated display:
Spatially distributed

Separated display:

spatially stacked

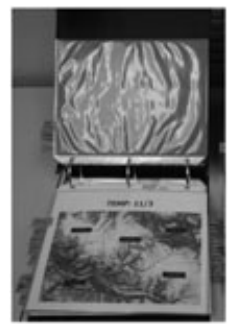

Images adapted from Information Design Journal, Displaying multi-domain graphical database information, 11(1), 2002, Kroft, P. D., \& Wickens, C. D., p.48, Figure 2. With kind permission by Springer Science+Business Media; Educational Psychology Review, Cognitive architecture and instructional design, 10(3), 1998, Sweller, J., van Merriënboer, J. J. G., \& Paas, F. G. W. C. p. 278, Figure 2. With kind permission by John Benjamins Publishing Company, Amsterdam/ Philadelphia. www.benjamins.com

presenting complex weather displays one at a time versus with a mapwall presenting the same maps printed out from the computer and posted along a wall. Participants in the computer condition could use animations and map comparison tools commonly used by forecasters to improve forecasting. Yet participants in the mapwall condition made predictions more than $50 \%$ faster than people in the computer condition, with no drop in accuracy.

One alternative explanation involves the potentially harmful effects of animation (Mayer, Griffith, Jurkowitz, \& Rothman, 2008; Mayer \& Moreno, 2003; Tversky, Morrison, \& Betrancourt, 2002). However, an unpublished study removing animations replicated the time benefit of the distributed layout (Jang, 2009). Another alternative explanation relates to affordances of paper versus computers (Dillon, 1992), but past replications of the effect with only computers rules out this possibility (Anderson, Hill, Parkin, \& Garisson, 2007; Wiley, 2001). A third possibility and the main theoretical focus of the current study is that the time effect was caused by the physical layout per se.

\section{The memorization strategy hypothesis}

A number of researchers have suggested that people choose problem solving strategies that reduce information retrieval costs (Ballard, Hayhoe, \& Pelz, 1995; Fu \& Gray, 2000; Fu \& Gray, 2006; Gray \& Fu, 2004; Gray, Schoelles, \& Sims, 2004). When information is in the world and the cost of accessing external information is low, people do not memorize information and instead use a perceptual-motor strategy (e.g. turning one's head or eyes when needed). In contrast, as the access-cost of external information increases, information in the head becomes relatively more accessible and people tend to use a memorization strategy. Fu and Gray (2000) examined the effects of imposing a 1-second delay in how quickly source information was revealed after key press in a copying task. They found that the 1-second delay led participants to take extra time to memorize information rather than repeatedly consult the source display.

Applying the information access cost construct to distributed/stacked displays, presumably distributed displays creates a lower-cost external information environment rela- 
tive to stacked displays because information is only a head turn away versus a page turn away. Under this account, those with a stacked display will invest time memorizing information, which would produce longer task times.

The goal of this article is to examine the time effect of spatial layout in a robust experimental design with an emphasis on testing an underlying mechanism that involves the trade-off between memorization and external information reliance (studies 1 and 2). The underlying memorization mechanism was tested using a surprise memory test for recently processed task information content.

\section{STUDY 1}

The goals of this experiment were to (i) establish whether the time benefit is specific to information integration tasks and (ii) obtain evidence for cognitive processing differences between display formats (distributed versus stacked) and task types (integration versus non-integration), especially in terms of memory. For non-integrative tasks, there should be no difference in memory between the display formats, because each information page stands alone and has no need to be integrated.

\section{Methods}

\section{Participants}

Eighty-three undergraduates (41 women and 42 men) at the University of Pittsburgh participated for course credit.

\section{Materials}

In the integration task, the problem was designed to require integration of information across pages. To produce a final answer, participants had to process information on each page, mentally note or write down intermediate results on provided blank sheets, and integrate those notes in the end. By contrast, the non-integration task did not require any information integration; each problem was complete by itself.

Integration task (deer task). The deer task asked participants to compute the number of surviving adult deer and fawns (of 100 for each) in each of five regions by applying prediction rules. Participants were told that deer die when they lose too much weight from extreme temperatures (i.e. heat and cold stress) and lack of food (i.e. snow depths more than 7 inches disturb food searching). Participants saw rules in text with a summary diagram (see Appendix A for the detailed rules).

Following the rule pages, there were task information pages, each showing temperature or snow depth for five different regions, for 10 different days $(2$ pages/ day $\times 10$ days $=20$ pages in total). Thus, including the four pages summarizing the problem solving rules, there were 24 pages that could be accessed during problem solving. Temperature and snow depth were presented in a paired manner for each day (Figure 1). Predictions were to be made separately for adults and fawns in each region. Participants needed to make 10 predictions ( 5 regions $\times 2$ predictions, one for adults and one for fawns) about the total weight loss summed across days.
Non-integration task (symbol task). A symbol arithmetic task was adapted from the study by Blessing and Anderson (1996), to roughly match the integration task on basic features (number of pages, operations per page, and rules to use; page organization; task difficulty) but involve no integration across pages. As with the deer task, rules were presented on the first page, and 20 pages of arithmetic problems followed. There were five rules, roughly matched to the rules in the deer task. The first four rules defined four different symbols as arithmetic operators; $\mathrm{A} \square \mathrm{B}=\mathrm{A} \times \mathrm{B}+5, \mathrm{~A} \triangle \mathrm{B}=(\mathrm{A}-\mathrm{B}) / 3$, $\mathrm{A} \odot \mathrm{B}=(\mathrm{A}+\mathrm{B}) \times 7, \mathrm{~A} \star \mathrm{B}=\mathrm{A} / \mathrm{B} \times 9$. The fifth rule described a decision criterion used to judge which problems should be solved and which problems should be skipped: 'Only solve problems if one number is greater than or equal to 10'. To match the task load in the deer task, each page involved five problems all having a shared symbol operator, so that only one operator rule was used per page. Problems were selected such that the same number of problems was solved per page as weight loss applications in the deer task.

Memory test. After completing the problem solving task, participants were given a surprise memory tasks that asked them to recall all information seen in the last four pages of the given task: (i) temperatures and snow depths in each region for the last 2 days in the integration task and (ii) all the symbol problems they saw, regardless of being solved or not, for the last four pages in the non-integration task.

\section{Design}

A $2 \times 2$ between subjects factorial design crossing type of spatial layout (stacked versus distributed) with type of task (integration versus non-integration) was used. In the stacked condition, information pages were attached to cardboard and put in a binder to prevent participants from seeing information on other pages (see Figure 1). In the integration task, pages were ordered by time, and indexes on each page showed page number and date. In the non-integration task, indexes showed page numbers.

In the distributed condition, pages of information were attached to a wall in a distributed manner, using a left-toright, top-to-bottom order in a $6 \times 4$ display. The four rule pages were in the left-most column. The 20 task pages were organized as five sets in the upper panel $(5 \times 2)$ and five sets in the lower panel $(5 \times 2)$. Note that how each page differed depends on high-spatial frequency details not accessible in peripheral vision.

\section{Procedure}

First, participants received training and practice on the task to which they were randomly assigned, practicing with the computer as a relatively neutral training method across conditions, but perhaps with a conservative bias toward the stacked condition. Participants went through example task pages with an experimenter and tried to apply the rules until they could use the rules correctly. To assist these calculations and to make participant solution methods visible, a clipboard and paper were provided.

Second, participants worked on the assigned task. For 30 participants (10 in each integration condition and 5 in each non-integration condition), the experimenter recorded 

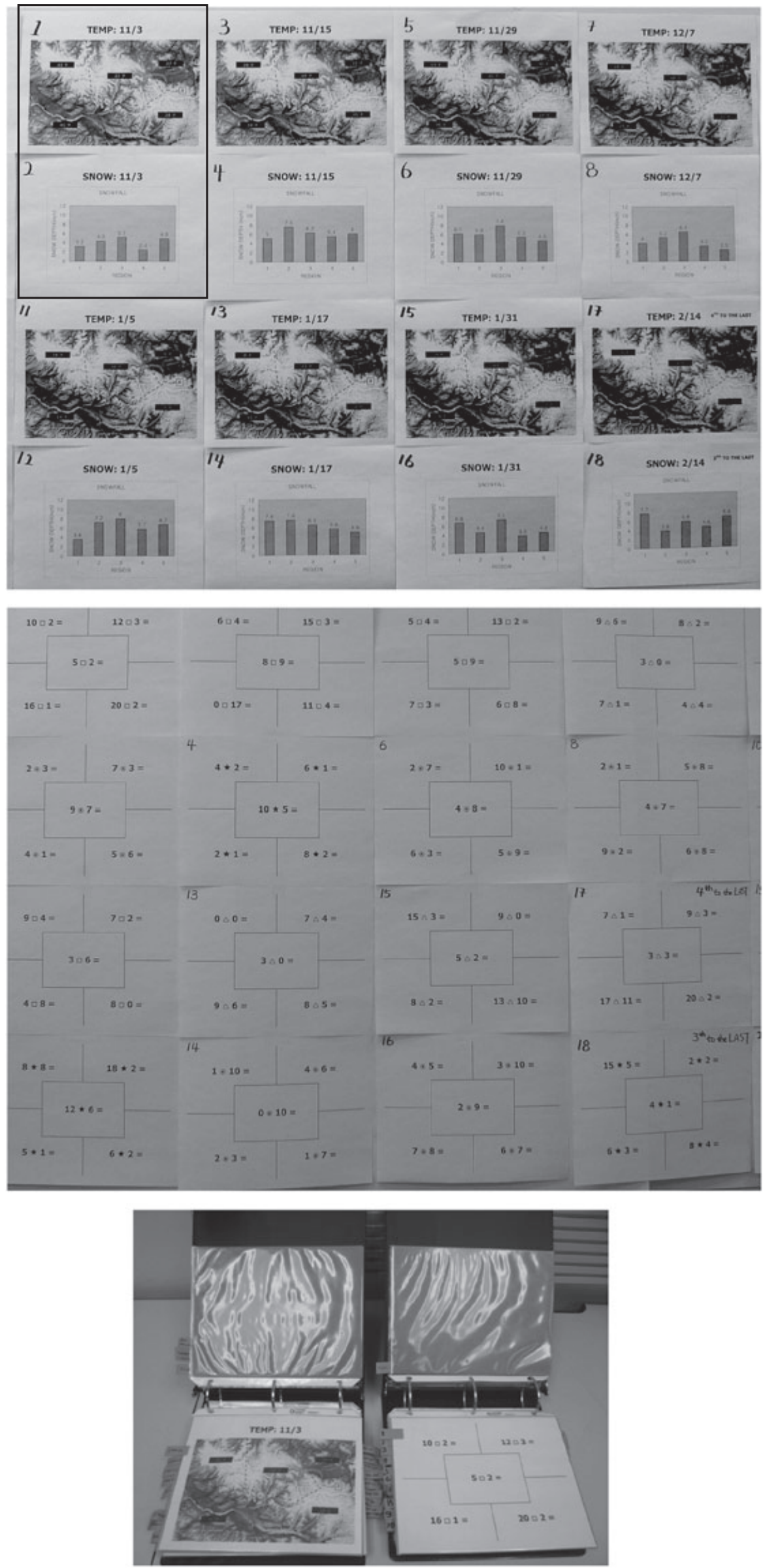

Figure 1. The experimental conditions: distributed-integration (top panel; magnified figure for the integration task on the right), distributedintegration (middle panel), stacked-integration (bottom panel left), and stacked-non-integration (bottom panel right) 
returns to each page by hand. In the distributed display condition, the page referral behavior was assessed by observing participants' eye, head, and body movement; because the distributed display occupied an area of $34^{\prime \prime} \times 66^{\prime \prime}$ of a wall, people had to walk along the display. Also, many participants often pointed at the page being examined.

Finally, participants were asked to turn away from the task information and complete a memory task, which consisted of task pages with blanks at the location where items of information were originally placed.

\section{Results and discussion}

The five participants who failed to finish the practice or main task within two standard deviations above the mean were excluded from analyses: four from the integration task and one from the non-integration task. This left 78 participants: 39 in the integration task condition (20 women) and 39 in the non-integration task condition (18 women).

\section{Time and accuracy: effects of spatial layout within each task type}

The time spent finishing the task was submitted to an analysis of variance with two factors: type of spatial layout (distributed versus stacked) and type of task (integration versus non-integration). The means and standard deviations for task time and accuracy of the integration and nonintegration task are presented in Table 2.

A large distributed display time advantage was observed, which confirmed that the previously observed effect was not specific to a contrast between information on a computer monitor versus on distributed sheets of paper. However, the time effect was observed only in the integration task, $F(1,38)=32.7, p<.001$, Cohen's $d=1.8$. Participants in the stacked condition spent about 6 more minutes to finish the task than participants in the distributed condition. By contrast, in the non-integration task, participants in distributed and stacked display conditions spent similar amounts of time to solve the symbol arithmetic problems, $F(1,38)=0.00, p=.98$, Cohen's $d=0.01$. The accuracies of both layout conditions were the same within both types of task, $F(1,38)=0.39, p=.54$, Cohen's $d=0.19$.

Next, we counted how many times participants returned to the rule pages. Remembering rules is necessary throughout the task, whereas temperature/snow information details can be discarded once they were processed and their results integrated into the working totals. Thus, only the memory test directly measures the kind of incidental task memory of focus in the memorization hypothesis. However, as load increases in the stacked condition due to memorization, a secondary consequence could be forgetting of other critical task details, like task rules. We would only expect this effect to show in the integration task where information layout causes a cognitive load from information integration.

In the integration task, participants using the stacked display went back to the rule page much more frequently $(M=13.3)$ than did participants using the distributed display $(M=3.8), F(1,15)=6.2, p=.03$, Cohen's $d=1.36$. In the nonintegration task, there was a non-significant yet sizable difference between the distributed $(M=11.4)$ and stacked $(M=15.2)$ display conditions, $F(1,9)=3.9, p=.08$, Cohen's $d=1.25$; the lack of statistical significance with such a large effect size can be attributed to the low $n$. If there is a replicable effect of display format for both task types (as these results suggest), then such an effect could be taken as consistent with the memorization hypothesis in that participants had to engage in more memorization (as measured by relooks) in the stacked condition, presumably because, remembering the rules to apply is an integrative element of both tasks. Note that early phases of problem solving often rely on example memories for the procedural elements of the task (Anderson, Fincham, \& Douglass, 1999), predicting that display format effects for rules disappear with task practice. In any case, the display effect on rule page references was not large enough to explain the huge effect on overall task time, as each rule page reference was typically on a few seconds.

\section{Memory test}

For the integration task, because of the large difference in time lag across display conditions between seeing the last pages and the memory test execution, memory test results were not analyzed in this study but will be examined in Study 2. For the non-integration task, which had no time lag confound between stacked and distributed conditions, a score for correctly recalled information was computed according to the following coding scheme. There were three pieces of information to remember (i.e. left operand, operator, and right operand), but a problem was counted as a whole as one point because the same symbol was used for all problems within a page.

Consistent with our hypothesis, there was no difference observed $[F(1,37)=1.9, p=.18]$ in terms of recall in the non-integration task. Overall, non-integration tasks simply present a basic forgetting function of information processed once and quickly forgotten (recall rates of $2 \%, 2 \%, 1 \%$, and $24 \%$ across the last four pages), and spatial layout per se conferred no special memory benefit.

\section{STUDY 2}

Incidental memory in the distributed versus stacked conditions with the integration task could not be cleanly examined

Table 2. Study 1: means and standard deviations for practice time, task time, and task accuracy of integration and non-integration tasks

\begin{tabular}{|c|c|c|c|c|c|c|c|c|}
\hline & \multicolumn{4}{|c|}{ Integration (deer task; $n=39$ ) } & \multicolumn{4}{|c|}{ Non-integration (symbol task; $n=39$ ) } \\
\hline & \multicolumn{2}{|c|}{ Distributed $(n=18)$} & \multicolumn{2}{|c|}{ Stacked $(n=21)$} & \multicolumn{2}{|c|}{ Distributed $(n=20)$} & \multicolumn{2}{|c|}{ Stacked $(n=19)$} \\
\hline & $M$ & $S D$ & $M$ & $S D$ & $M$ & $S D$ & $M$ & $S D$ \\
\hline Task time (minutes) & 10.6 & 2.7 & 16.8 & 4 & 8.3 & 1.6 & 8.3 & 1.5 \\
\hline Task accuracy (\%) & 75.5 & 16.5 & 79 & 13.3 & 95.7 & 3.5 & 94.9 & 5.0 \\
\hline
\end{tabular}


in Study 1 because of the confound of layout condition with large lag condition differences. Study 2 addresses this point by moving the memory test to immediately before participants reached the final information page.

Overall, Study 2 was identical to Study 1 except that (i) the memory test was given to participants immediately after they finished processing the second-to-last page (i.e. page 19) and (ii) only the integration task was used this time.

\section{Methods}

\section{Participants}

Forty-one undergraduates (23 women and $18 \mathrm{men}$ ) at the University of Pittsburgh participated for course credit.

\section{Design and procedure}

All participants solved the integration (deer) task in either stacked or distributed layouts. The problem-solving phase was interrupted with a memory test, given immediately after participants finished processing the second-to-last page. The second-to-last rather than last page was used because it was expected to be difficult to pinpoint the precise moment when participants finish processing the last page. The memory test consisted of four pages taken from pages 16 to 19. Following the memory test, participants completed the task as in Study 1.

\section{Results and discussion}

Five participants who failed to obtain at least $50 \%$ accuracy and/or failed to finish the task within two standard deviations above the mean were discarded as outliers. Two were poor at calculation. Another three participants could not solve the task on their own despite abundant practice. In total, 33 participants were included in analyses with 16 in the distributed condition (11 women) and 17 in the stacked condition (9 women).

\section{Time and accuracy}

Problem solving time was submitted to an analysis of variance with the factor of type of spatial layout (distributed versus stacked). The means and standard deviations for task time and accuracy in the integration task by the type of spatial layout are presented in Table 3. Despite the interruption of a memory test, the time benefit for the distributed format was obtained again: participants with the distributed display $(M=10, S D=3.3)$ finished the task about 5 minutes faster than participants with the stacked display $(M=14.7, S D=6), F(1,32)=7.9, p=.009$, Cohen's $d=1.0$. Accuracy $[F(1,32)=2.74, p=.11]$ again showed no effect of display format.

Table 3. Study 2: means and standard deviations for practice time, task time, and task accuracy of integration task

\begin{tabular}{|c|c|c|c|c|}
\hline \multirow[b]{4}{*}{ Task time (minutes) } & \multicolumn{4}{|c|}{ Integration (deer task) } \\
\hline & \multicolumn{2}{|c|}{$\begin{array}{c}\text { Distributed } \\
\quad(n=16)\end{array}$} & \multicolumn{2}{|c|}{$\begin{array}{l}\text { Stacked } \\
(n=17)\end{array}$} \\
\hline & $M$ & $S D$ & $M$ & $S D$ \\
\hline & 10.0 & 3.3 & 14.7 & 6.0 \\
\hline Task accuracy $(\%)$ & 76.8 & 13.0 & 83.5 & 10.0 \\
\hline
\end{tabular}

\section{Memory test}

A score for correctly recalled information was computed using the following coding scheme. Each correct value written anywhere within the page was given one point. A correct value in the correct location was given two points, resulting in a theoretical maximum of 40 total points (5 regions $\times 4$ pages $\times 2$ points). However, no participant correctly placed a correct value in the correct location, simplifying the coding scheme to one point per correct value within the given page (20 points maximum).

We predicted participants using the stacked display would remember more items than participants using the distributed display. There was no overall main effect across all pages. Participants in the stacked condition $(M=15.3 \%, S D=7.8 \%)$ seems to recall only slightly more items overall than participants in the distributed condition $(M=13.1 \%, S D=9.1 \%)$, but this overall difference was not statistically significant, $F(1,32)=1.4, p=.25$. The low recall on items in pages 16 and 18, which provided snow depth information, can be explained by the nature of the content on the pages; the key information the problem solvers needed to extract was whether any snow depth bar in any region is over a threshold (i.e. 7-inch) or not. Thus, it was not necessary to encode the actual numbers of snow depth inch on each bar in detail, and we can exclude the recall data from these pages as floor effects. The $t$-tests (two-tailed) for each of the two temperature pages showed a significant difference in recall on page 17; participants with stacked display recalled more than participants with distributed display did (33\% versus $19 \%), t(32)=2.2, p=.04$, Cohen's $d=0.7$. Furthermore, the memory data provide some additional evidence of incidental memory as a function of being an integration task with a lack of the recency effect that was found in the nonintegration task; instead, there was better memory for older items (25\% on page 17) compared with more recent (15\% on page 19 ) items. This condition incidental memory difference, especially for content that was probably revisited multiple times, provides indirect support for the memorization hypothesis.

\section{GENERAL DISCUSSION}

Studies 1 and 2 consistently showed a large time advantage of distributed displays over stacked displays. Study 1 demonstrated that the speedup effect is specific to integrative tasks, with no effect on a non-integrative task such as simple arithmetic. Study 2 results were also suggestive that integrative tasks induce different cognitive processes from non-integrative tasks in terms of memory consequences. Although participants who solved a non-integrative task showed a typical recency effect in the serial position memory curve (Study 1), those who solved an integrative task showed a reversed-recency effect (Study 2). There were also display condition effects on rule memory.

Given the complexity of the task and cognitive activities involved, it is unlikely that a single factor alone accounts for the whole effect. The overall time effect may then have been produced by a combination of memorization time, higher level of rule referring behavior (cost of manual interactivity), and 
cost of concurrent non-visual tasks. First, the memorization strategy hypothesis (i.e. when to-be-integrated information is stacked, people invest time to memorize and encode the information into internal memory to reduce the external information retrieval cost) was partially supported by the memory data in Study 2. Participants in the stacked condition appeared to remember more to-be-integrated information than those in the distributed condition, particularly for information that had to be symbolically encoded and retrieved. This memory data pattern is a clue that participants using the stacked display may have invested more effort to encode the information and the evidence of that effort was better incidental memory.

Second, cost of manual interactivity might also play a small role. Clearly, participants with the stacked display had to turn a page to move onto the next one: at least 23 pageturns are required to reach to the last page, and sometimes, they returned to the rule pages. This cost is, however, too small to explain the whole 6-minute difference between conditions; it can only explain about a 1-minute difference at the most if we suppose that page-turning consumes a second and a participant processes all pages twice.

Finally, the cost of concurrent non-visual tasks may also contribute to the effect. The integration task used in this research does not explicitly involve a separate secondary task. However, it may be that the stacked display can be thought to increase an implicit secondary task of keeping track of information pages; such activities could consume some cognitive resources. A similar study done in an educational domain found that students who worked with stacked instructional materials reported relatively higher cognitive load than those who worked with distributed instructions (Jang, Schunn, \& Nokes, 2011). This hypothesis should be explored in future research so as to further unpack the causes of differing levels of cognitive load.

\section{Caveats}

The interplay of several plausible explanations in response to differing access-costs across formats provided some insights into explaining the time difference in problem solving, but our current top candidate cause for the effect, the memorization strategy selection hypothesis, was only indirectly supported by inference from (uneven) memory results. Future research should examine more direct measures of memorization processes. Given that the task information mainly consisted of numbers (temperatures and snow inches) and the exact values of many numbers become obsolete once they were identified as not relevant to the final answer or not, the indirect and temporally distant measure of memory retrieval might not be sensitive enough to show differences in memorization processes. Potentially, eye-tracking methods would help resolve this issue. Displays with high access cost and cognitive load should lead to longer fixation times.

\section{Theoretical and practical implications}

This speedup effect is theoretically surprising when viewed in light of the combination of the human cognitive architecture: limits of visual acuity and limited visual working memory capacity. Detailed visual input processing is only possible within the small center of fovea (De Valois
\& De Valois, 1988; Hirsch \& Curcio, 1989), and thus, participants could not be able to perceive in the periphery the fine details that they were asked process in our task (e.g. small numbers). In addition to the issue of visual acuity, the capacity of visual working memory is also limited to only three or four objects (Baddeley, 2003). Thus, even when the two pages are laid out side-by-side, items seen on one page will slip out of one's perception and visual working memory as one moves on to the next page. In theory, this nature of visual perception makes distributed and stacked formats equivalent vis-à-vis information immediately accessible to the problem solver. Performance differences must come from more indirect memory or strategy differences such as with the memorization strategy hypothesis.

In addition to theoretical implications, the advantages of the distributed format can be applied to productivity in many workplace settings. The current studies and work from other laboratories have found a strong advantage for distributed formats (benefit of dual or a large monitor: Anderson et al., 2007), suggesting that multiple and larger display settings should be generally recommended. Furthermore, as claimed by proximity compatibility theorists, a single integrated display that combines too many information sources could hurt performance by increasing information search time because of visual clutter. Comparing with a single integrated display, a distributed display has a chunking advantage: each information source is a meaningful chunk that contains consistent information that might reduce search time when the number of to-be-integrated information sources is more than two.

\section{Future work}

There are a number of remaining questions to be answered. Beside the underspecified mechanism, there is an additional human factor question or user choice question: why do people prefer a single window setting, which is not optimal display for integrative tasks? In the studies by both Wiley (2001) and Anderson et al. (2007), people initially preferred a single monitor setup and refused the multiple window browsers or dual monitor setting when given the choice. Because implementing research findings in practice often faces unexpected resistance, this seemingly odd preference for a single monitor must be examined further.

Finally, expertise can be an important factor to consider (Kalyuga, Ayres, Chandler, \& Sweller, 2003). The meteorologists, who offered the starting point of this research, were intermediate weather forecasters, and the students who participated in this research were all novices in terms of the specific task (i.e. the deer task and the symbol task). Thus, no study has examined the distributed display time advantage in experts. To effectively apply these findings in real world, a study of the effect across levels of expertise should be conducted.

\section{REFERENCES}

Anderson, J., Fincham, J. M., \& Douglass, S. (1999). Practice and retention: A unifying analysis. Journal of Experimental Psychology: Learning, Memory, and Cognition, 25, 1120-1136.

Anderson, J., Hill, J., Parkin, P., \& Garisson, A. (2007). Productivity, screens, and aspect ratios; A comparison of single, traditional aspect, dual, traditional aspect and single, widescreen aspect computer displays 
over simulated office tasks across performance and usability. Illinois: NEC Display Solutions.

Baddeley, A. (2003). Working memory: Looking back and looking forward. Nature Reviews Neuroscience, 4, 829-839.

Ballard, D. H., Hayhoe, M. M., \& Pelz, J. B. (1995). Memory presentations in natural tasks. Journal of Cognitive Neuroscience, 7, 66-80.

Blessing, S., \& Anderson, J. R. (1996). How people learn to skip steps. Journal of Experimental Psychology: Learning, Memory, and Cognition, 22, 576-598.

De Valois, R. L., \& De Valois, K. K. (1988). Spatial vision. New York: Oxford University Press.

Dillon, A. (1992). Reading from paper versus screens: A critical review of the empirical literature. Ergonomics, 35, 1297-1326.

Fu, W. T., \& Gray, W. D. (2000). Memory versus perceptual motor tradeoffs in a blocks world task. Paper presented at the Proceedings of the Twenty-second Annual conference of the Cognitive Science Society, Hillsdale, NJ.

Fu, W. T., \& Gray, W. D. (2006). Suboptimal tradeoffs in information seeking. Cognitive Psychology, 52, 195-242. doi: 10.1016/j.cogpsych.2005.08.002

Gray, W. D., \& Fu, W. T. (2004). Soft constraints in interactive behavior: The case of ignoring perfect knowledge in-the-world for imperfect knowledge in-the-head. Cognitive Science: A Multidisciplinary Journal, 28, 359-382.

Gray, W. D., Schoelles, M. J., \& Sims, C. R. (2004). Learning to choose the most effective strategy: Explorations in expected value. Paper presented at the Proceedings of the sixth International Conference on Cognitive Modeling, Pittsburgh, PA: Carnegie Mellon University/University of Pittsburgh.

Hirsch, J., \& Curcio, C. A. (1989). The spatial resolution capacity of human foveal retina. Vision Research, 29, 1095-1101.

Jang, J. (2009). Lost in space: The surprising role of information spatial layout. Master of Science, University of Pittsburgh, Pittsburgh, PA.

Jang, J., Schunn, C. D., \& Nokes, T. J. (2011). Spatially distributed instructions improve learning outcomes and efficiency. Journal of Educational Psychology, 103, 60-72. doi: 10.1037/a0021994

Kalyuga, S., Ayres, P., Chandler, P., \& Sweller, J. (2003). The expertise reversal effect. Educational Psychologist, 38, 23-31. doi: 10.1207/ S15326985EP3801_4

Kroft, P. D., \& Wickens, C. D. (2002). Displaying multi-domain graphical database information. Information Design Journal, 11, 44.

Mayer, R. E., Griffith, E., Jurkowitz, I. T. N., \& Rothman, D. (2008). Increased interestingness of extraneous details in a multimedia science presentation leads to decreased learning. Journal of Experimental Psychology. Applied, 14, 329-339.

Mayer, R. E., \& Moreno, R. (2003). Nine ways to reduce cognitive load in multimedia learning. Educational Psychologist, 38, 43-52.

Sweller, J., \& Chandler, P. (1994). Why some material is difficult to learn. Cognition and Instruction, 12, 185-233. doi: 10.1207/s1532690xci1203
Sweller, J., van Merriënboer, J. J. G., \& Paas, F. G. W. C. (1998). Cognitive architecture and instructional design. Educational Psychology Review, 10, 251-296. doi: 10.1023/A:1022193728205

Trafton, G., Trickett, S., Schunn, C., \& Kirschenbaum, S. (2007). Complex visualizations, spatial transformations, and uncertainty. Presented at the meeting of the Naval Research Laboratory, Washington, DC.

Tversky, B., Morrison, J., \& Betrancourt, M. (2002). Animation: Can it facilitate? International Journal of Human Computer Studies, 57, 247-262. van Merriënboer, J. J. G., \& Sweller, J. (2005). Cognitive load theory and complex learning: Recent developments and future directions. Educational Psychology Review, 17, 147-177. doi: 10.1007/s10648-005-3951-0

Wickens, C. D., \& Carswell, C. M. (1995). The proximity compatibility principle: Its psychological foundation and relevance to display design. Human Factors, 37, 473(422). doi: 10.1518/001872095779049408

Wickens, C. D., \& McCarley, J. S. (2008). Applied attention theory. Boca Raton, FL: Taylor \& Francis.

Wiley, J. (2001). Supporting understanding through task and browser design. Paper presented at the Proceedings of the Twenty-third annual Conference of the Cognitive Science Society.

\section{APPENDIX A \\ PREDICTION RULES FOR INTEGRATION (DEER) TASK IN FORMULAE}

(1) Temperature weight loss (TempLoss):

IF temperature $\geq 30 \mathrm{~F}$, THEN adults lose $3 \mathrm{lb}$ (fawns lose $5 \mathrm{lb}$ )

(2) Temperature weight loss (TempLoss):

IF temperature $\leq-4 \mathrm{~F}$, THEN adults lose $5 \mathrm{lb}$ (fawns lose $7 \mathrm{lb}$ )

(3) Snow depth weight loss (SnowLoss):

IF snow depth $\geq 7$ inches, THEN adults lose $7 \mathrm{lb}$ (fawns lose $10 \mathrm{lb})$

(4) Total weight loss

IF $\sum_{\text {page }=1}^{n}\left(\right.$ TempLoss $_{\text {page }}+$ SnowLoss $\left._{\text {page }}\right) \geq 30 \mathrm{lb}$, THEN adults' population becomes 80 (fawns' becomes 60)

(5) Total weight loss:

IF adults' population is 80 (fawns' is 60), for every $\sum_{\text {page }=n+1}^{m}\left(\right.$ TempLoss $_{\text {page }}+$ SnowLoss $\left._{\text {page }}\right) \geq 10 \mathrm{lb}$, THEN population cuts down by half 